\title{
SYSTEMATIC BLUESHIFT OF LINE PROFILES IN THE TYPE IIn SUPERNOVA 2010j1: EVIDENCE FOR POST-SHOCK DUST FORMATION?
}

\author{
Nathan Smith ${ }^{1}$, Jeffrey M. Silverman ${ }^{2}$, Alexei V. Filippenko ${ }^{2}$, Michael C. Cooper ${ }^{3}$, Thomas Matheson ${ }^{4}$, Fuyan Bian $^{1}$, \\ Benjamin J. WeineR ${ }^{1}$, AND Julia M. COMERFORD ${ }^{5}$ \\ ${ }^{1}$ Steward Observatory, University of Arizona, 933 North Cherry Avenue, Tucson, AZ 85721, USA; nathans @ as.arizona.edu \\ ${ }^{2}$ Department of Astronomy, University of California, Berkeley, CA 94720-3411, USA \\ ${ }^{3}$ Department of Physics and Astronomy, University of California, 4129 Frederick Reines Hall, Irvine, CA 92697-4575, USA \\ ${ }^{4}$ National Optical Astronomy Observatory, 950 North Cherry Avenue, Tucson, AZ 85719-4933, USA \\ ${ }^{5}$ Astronomy Department, University of Texas, Austin, TX 78712, USA \\ Received 2011 August 14; accepted 2011 October 3; published 2011 December 9
}

\begin{abstract}
Type IIn supernovae ( $\mathrm{SNe}$ ) show spectral evidence for strong interaction between their blast wave and dense circumstellar material (CSM) around the progenitor star. SN 2010jl was the brightest core-collapse supernova in 2010, and it was a Type IIn explosion with strong CSM interaction. Andrews et al. recently reported evidence for an infrared (IR) excess in SN 2010jl, indicating either new dust formation or the heating of CSM dust in an IR echo. Here we report multi-epoch spectra of SN 2010jl that reveal the tell-tale signature of new dust formation: emission-line profiles becoming systematically more blueshifted as the red side of the line is blocked by increasing extinction. The effect is seen clearly in the intermediate-width (400-4000 $\mathrm{km} \mathrm{s}^{-1}$ ) component of $\mathrm{H} \alpha$ beginning roughly 30 days after explosion. Moreover, we present near-IR spectra demonstrating that the asymmetry in the hydrogen-line profiles is wavelength dependent, appearing more pronounced at shorter wavelengths. This evidence suggests that new dust grains had formed quickly in the post-shock shell of SN 2010jl arising from CSM interaction. Since the observed dust temperature has been attributed to an IR echo and not to new dust, either (1) IR excess emission at $\lambda<5 \mu \mathrm{m}$ is not a particularly sensitive tracer of new dust formation in $\mathrm{SNe}$, or (2) some assumptions about expected dust temperatures might require further study. Lastly, we discuss one possible mechanism other than dust that might lead to increasingly blueshifted line profiles in SNe IIn, although the wavelength dependence of the asymmetry argues against this hypothesis in the case of SN 2010jl.
\end{abstract}

Key words: circumstellar matter - dust, extinction - stars: evolution - stars: mass-loss - stars: winds, outflows supernovae: general

Online-only material: color figures

\section{INTRODUCTION}

The formation of dust grains in core-collapse supernova ( $\mathrm{SN}$ ) explosions is of interest in part because SNe contributed to the dust budget of the early interstellar medium seen in highredshift galaxies. SNe that may arise from very massive stars are of particular importance in this regard (e.g., Cherchneff \& Dwek 2010).

New dust formation in an SN may give rise to three observable signatures seen concurrently: (1) infrared (IR) excess due to thermal emission from hot or warm dust, (2) increased rate of fading of the optical flux (typically on the radioactive decay tail), and (3) progressive and systematic blueshift of emission-line profiles when the receding parts of the ejecta are increasingly blocked by new dust. The first two might also arise from an IR echo due to pre-existing dust in the circumstellar material (CSM) or from the escape of radioactive luminosity due to decreasing optical depth in the SN ejecta, respectively, but the presence of all three concurrently is generally taken to signify that these effects are caused by the formation of new dust grains. Clear observational evidence of all three effects, however, is rare, limited to cases of very nearby SNe. In addition to the first well-established case of SN 1987A (Danziger et al. 1989; Lucy et al. 1989; Gehrz \& Ney 1989; Wooden et al. 1993; Colgan et al. 1994), only a few other compelling examples of dust formation in normal SN ejecta have been found. Thus far, though, dust masses estimated for normal core-collapse $\mathrm{SNe}$ $\left(M_{\text {ZAMS }}<20 M_{\odot}\right.$ progenitor stars $)$ are very low, being $1-3$ orders of magnitude too small to account for the dust budget inferred in high-redshift galaxies (see, e.g., Elmhamdi et al. 2003; Sugerman et al. 2006; Meikle et al. 2007, 2011; Kotak et al. 2009; Andrews et al. 2010; Szalai et al. 2011). In these $\mathrm{SNe}$, the dust formation occurs in the rapidly expanding $\mathrm{SN}$ ejecta a few hundred days after explosion.

Recent far-IR observations of SN 1987A have revealed an unexpectedly large amount of very cold dust in the SN ejecta (Matsuura et al. 2011). The $\sim 20 \mathrm{~K}$ dust is much cooler than expected, and the huge mass of $0.4-0.7 M_{\odot}$ is much more than the $10^{-4}$ to $10^{-3} M_{\odot}$ that had been inferred based on IR emission observed a few hundred days after the explosion (Wooden et al. 1993). It should be noted that the early mass estimates around $10^{-4} M_{\odot}$ were lower limits due to the fact that the dust may have resided in optically thick clumps, but the large mass detected recently in the far-IR nevertheless presents a significant mystery. If true, it implies that vast amounts of dust may somehow escape detection by IR emission when the dust is forming.

Recent evidence has been accumulating that a different mode of dust formation is also present in some $\mathrm{SNe}$, where dust grains can form efficiently and very early in the cold dense shell arising from strong CSM interaction. In this scenario, the high density in the radiative post-shock layers allows the post-shock gas to cool rapidly, thereby permitting dust formation. The first clear evidence for this mode of dust formation was seen in SN 2006jc, which showed all three effects described above. In addition to a near-IR excess from very hot dust and an increased fading rate, it showed a systematic blueshift in He I emission lines formed in 
the CSM interaction region (Smith et al. 2008b). In particular, Smith et al. (2008b) demonstrated that the relative strength of the systematic blueshift seen in He I lines at different wavelengths was consistent with the wavelength-dependent extinction one expects from small dust grains. This provided the first solid example of dust formed in the dense post-shock cooling zone of an SN dominated by CSM interaction, although it had been noted earlier as a possibility in the case of the SN IIn 1998S (Pozzo et al. 2004). Subsequently, Matilla et al. (2008) presented mid-IR data for SN 2006jc and additional spectra, yielding similar results.

SN 2006jc was a Type Ibn SN, and it is possible that C-rich SN ejecta crossing the reverse shock may have aided the efficiency of dust formation (see Smith et al. 2008b; Matilla et al. 2008). However, evidence for post-shock dust formation has also been reported in a few examples of Type IIn SNe that are not expected to have C-rich ejecta. These include SN $1998 \mathrm{~S}$ (Pozzo et al. 2004), SN 2005ip (Smith et al. 2009; Fox et al. 2009), and SN 2006tf (Smith et al. 2008a). More recently, some of the SNe IIn studied by Fox et al. (2011) also show possible evidence for dust formation, although these authors conclude based on the observed dust temperature that the bulk of the IR excess was probably due to an IR echo in most cases. Fox et al. (2011) provide a more detailed discussion of the issue of dust formation and IR echoes in SNe IIn. SNe IIn constitute only about 6\%-9\% of core-collapse SNe (Smith et al. 2011a; Li et al. 2011), so even if they are very efficient dust producers, it is not clear that they can account for the dust present in high-redshift galaxies unless they are more common at high redshift.

Andrews et al. (2011) recently reported an IR excess in the very bright Type IIn SN 2010jl. They note that the IR excess may arise from either newly formed dust or an IR echo from pre-existing dust, although they favor the hypothesis of an IR echo because of the observed dust temperature. In this paper we consider the evolution of optical emission-line profiles in SN 2010jl, which suggest the presence of newly formed dust as well.

SN 2010jl was discovered on 2010 November 3.52 (UT dates are used throughout this paper) by Newton \& Puckett (2010). It was intrinsically luminous, with a peak absolute magnitude of about -20 (Smith et al. 2011b). Early-time spectra showed that it is a Type IIn SN (Benetti et al. 2010; see also Filippenko 1997). In a previous paper (Smith et al. 2011b), we analyzed preexplosion archival images of the field of SN 2010jl obtained with the Hubble Space Telescope (HST), showing that the progenitor of SN 2010jl was likely to be a very massive star with an initial mass above $30 M_{\odot}$, and with dense CSM expanding at $40-120 \mathrm{~km} \mathrm{~s}^{-1}$ along our line of sight. Attributing the IR excess to pre-existing dust, Andrews et al. (2011) infer a very large amount of mass in the CSM of SN 2010j1, possibly suggesting a massive progenitor similar to luminous blue variables (LBVs). Additional information on the basic observed properties of SN 2010jl can be found in recent reports (Patat et al. 2011; Smith et al. 2011b; Andrews et al. 2011).

\section{OBSERVATIONS}

We obtained visual-wavelength spectra of SN 2010j1 on several epochs during the first $\sim 7$ months after discovery. The observations and data reduction for the two epochs of Keck spectra (2010 November 5 and 7) were already described in our previous paper (Smith et al. 2011b), where we also discussed the observations and data reduction for the first two epochs of spectra obtained with the Blue Channel spectrograph on the MMT (2010 November 5 and 6). The high-resolution
Table 1

Spectroscopic Observations of SN 2010j1

\begin{tabular}{lccccc}
\hline \hline Date & Tel./Inst. & Day $^{\mathrm{b}}$ & $\begin{array}{c}\delta \lambda^{\mathrm{c}} \\
(\AA)\end{array}$ & $\lambda / \Delta \lambda$ & $\begin{array}{c}W_{\mathrm{H} \alpha}{ }^{\mathrm{d}} \\
(\AA)\end{array}$ \\
\hline 2010 Nov. 5 & $\mathrm{K} / \mathrm{L}$ & 2 & $3250-7550$ & 1100 & 150 \\
2010 Nov 5 & $\mathrm{M} / \mathrm{B}$ & 2 & $6200-7500$ & 4500 & 133 \\
2010 Nov 6 & $\mathrm{M} / \mathrm{B}$ & 3 & $6200-7500$ & 4500 & 141 \\
2010 Nov 7 & $\mathrm{K} / \mathrm{D}$ & 4 & $4400-9520$ & 4400 & 188 \\
2010 Nov 15 & $\mathrm{L} / \mathrm{K}$ & 12 & $3400-10220$ & 600 & 217 \\
2010 Nov 30 & $\mathrm{L} / \mathrm{K}$ & 27 & $3400-10220$ & 600 & 297 \\
2011 Jan 4 & $\mathrm{L} / \mathrm{K}$ & 62 & $3400-10220$ & 600 & 519 \\
2011 Jan 16 & $\mathrm{M} / \mathrm{B}$ & 74 & $6200-7500$ & 4500 & 586 \\
2011 Jan 26 & $\mathrm{L} / \mathrm{K}$ & 84 & $3400-10220$ & 600 & 670 \\
2011 Feb 2 & $\mathrm{L} / \mathrm{K}$ & 91 & $3400-10220$ & 600 & 688 \\
2011 Feb 9 & $\mathrm{L} / \mathrm{K}$ & 98 & $3400-10220$ & 600 & 758 \\
2011 Feb 23 & $\mathrm{M} / \mathrm{F}$ & 112 & $8000-23000$ & 6000 & $\ldots$ \\
2011 Mar 31 & $\mathrm{L} / \mathrm{K}$ & 148 & $3400-10220$ & 600 & 1020 \\
2011 May 12 & $\mathrm{L} / \mathrm{K}$ & 190 & $3400-10220$ & 600 & 1120 \\
2011 Jun 27 & $\mathrm{M} / \mathrm{B}$ & 236 & $6300-7600$ & 4500 & 1350 \\
\hline
\end{tabular}

Notes.

a Telescope/Instrument: $\mathrm{K} / \mathrm{L}$ or $\mathrm{K} / \mathrm{D}=\mathrm{W}$. M. Keck Observatory with LRIS (Low-Resolution Imaging Spectrometer) or DEIMOS (Deep Imaging MultiObject Spectrograph); M/B = Multiple Mirror Telescope with the Blue Channel spectrograph; L/K = Lick Observatory $3 \mathrm{~m}$ Shane telescope and the Kast double spectrograph; $\mathrm{M} / \mathrm{F}=$ Magellan Observatory and the FIRE spectrograph.

b Days after discovery.

c Rest wavelengths.

${ }^{\mathrm{d}}$ Emission equivalent width of $\mathrm{H} \alpha$.

MMT spectra obtained on 2011 January 16 and June 27 are presented here for the first time, but the observational set-up and data reduction followed the same procedures as for the 2010 November MMT spectra described earlier (Smith et al. 2011b). We also present eight additional epochs of optical spectra obtained with the Kast double spectrograph (Miller \& Stone 1993) mounted on the Lick Observatory $3 \mathrm{~m}$ Shane telescope. All spectra were obtained with the long slit oriented at the parallactic angle (Filippenko 1982) to minimize losses caused by atmospheric dispersion. The spectral observations are summarized in Table 1. Most of the spectra are shown in Figure 1 (excluding the high-resolution MMT spectra which only covered a portion of the optical wavelength range), while Figures 2 and 3 illustrate details of the $\mathrm{H} \alpha$ line profile.

We also obtained one near-IR spectrum of SN 2010jl on 2011 February 23 using the Folded port IR Echellete (FIRE) spectrograph $^{6}$ mounted on the $6.5 \mathrm{~m}$ Baade telescope of the Magellan Observatory. We used FIRE in the high-resolution echellete mode with a slit width of $0^{\prime \prime} 6$, yielding a spectral resolving power of $\sim 6000$ or about $30 \mathrm{~km} \mathrm{~s}^{-1}$. Sky subtraction was accomplished by nodding along the slit in ABBA sequences, with a total on-source integration time of $20 \mathrm{~min}$. In this paper, we are primarily interested in comparing the line profiles of near-IR hydrogen lines to those of visual-wavelength hydrogen lines that exhibit asymmetry. Figure 4 shows the line profiles of $\mathrm{Pa} \beta$ and $\mathrm{Br} \gamma$ in the 2011 February 23 FIRE spectrum, compared to the $\mathrm{H} \alpha$ and $\mathrm{H} \beta$ profiles obtained a few weeks earlier.

\section{DISCUSSION}

\subsection{Results}

The main observational results of this paper are that (1) the $\mathrm{H} \alpha$ line profile of SN 2010jl provides a textbook example

\footnotetext{
6 http://web.mit.edu/ rsimcoe/www/FIRE/
} 


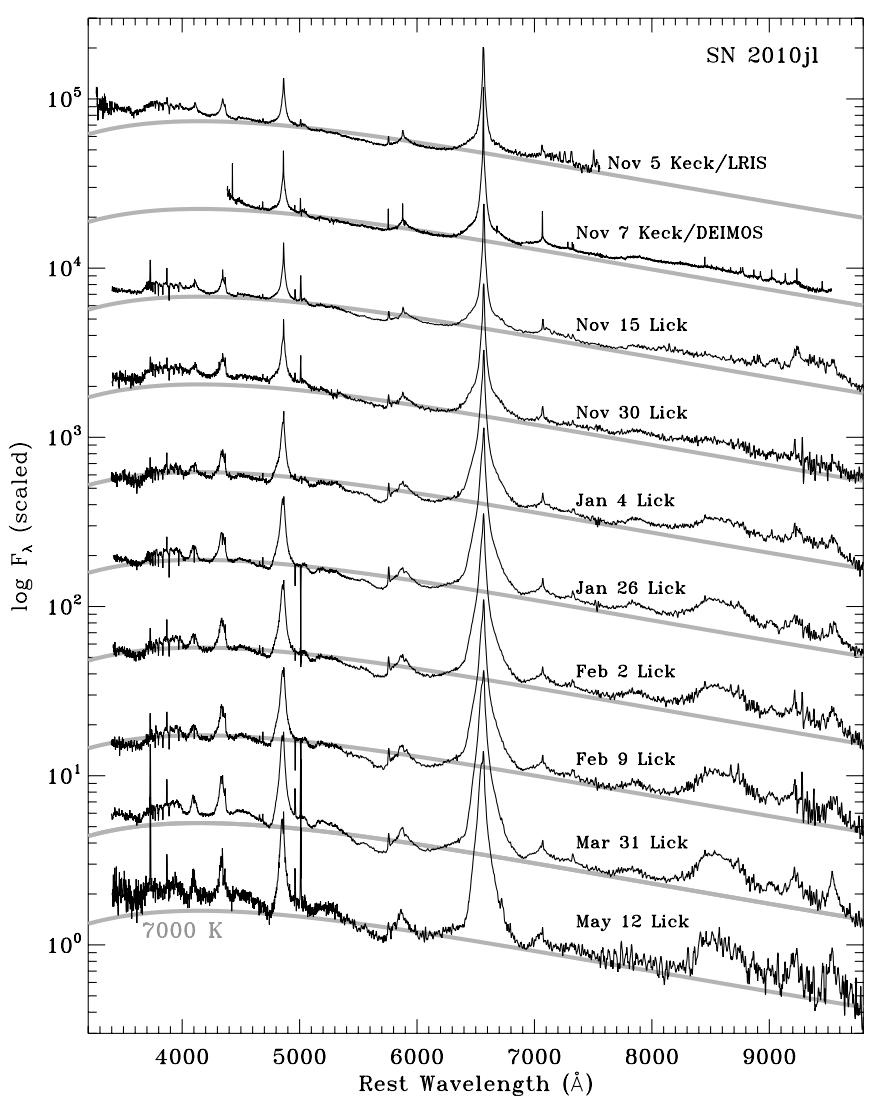

Figure 1. Optical spectra of SN 2010jl showing the spectral evolution from early times a few days post-discovery until 6 months later (see Table 1). Overall, the spectrum appears constant during this time period, with roughly the same $\sim 7000 \mathrm{~K}$ characteristic continuum temperature at these wavelengths. An important development, however, is the appearance of the Ca II IR triplet.

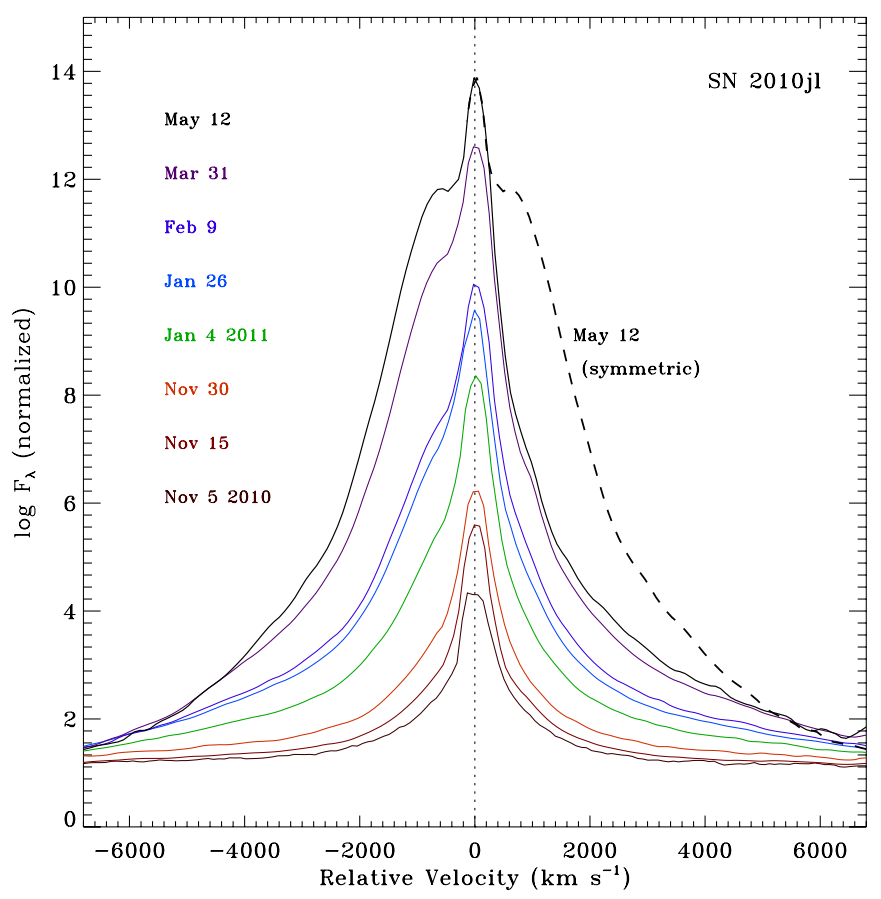

Figure 2. Evolution of the $\mathrm{H} \alpha$ profile of SN 2010j1, showing that the red side of the intermediate-width component weakens systematically compared with the blue side during the 6 months after discovery. The dashed curve shows the blue wing of the line reflected to the red side, demonstrating what the profile would look like if it were symmetric on the final epoch on May 12.

(A color version of this figure is available in the online journal.)

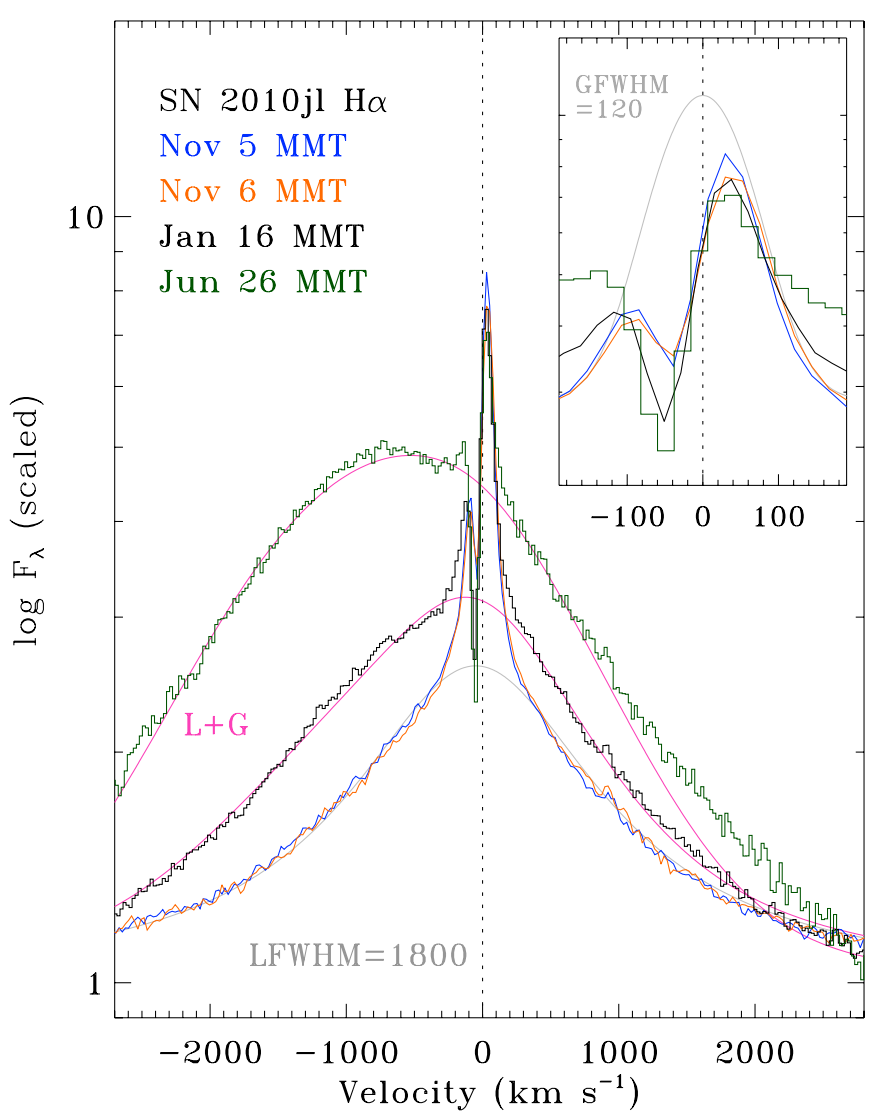

Figure 3. High-resolution spectra obtained with the MMT/Blue Channel showing the $\mathrm{H} \alpha$ line profile of SN 2010jl. All epochs used the same instrument configuration. This is similar to Figure 3 from Smith et al. (2011b), except that we have added two additional epochs of MMT spectra. The gray curve shows the same Lorentzian profile as in the previous paper, with a Lorentzian full width at half-maximum intensity (LFWHM) of $1800 \mathrm{~km} \mathrm{~s}^{-1}$. The magenta curves show the same Lorentzian profile plus a Gaussian shifted by $-700 \mathrm{~km} \mathrm{~s}^{-1}$ in both cases; the Gaussian component is obviously stronger for the magenta curve associated with the 2011 June 27 profile. The inset concentrates on the narrow $\mathrm{H} \alpha$ component from the CSM; here the gray curve is a symmetric Gaussian that is the same as in Smith et al. (2011b).

(A color version of this figure is available in the online journal.)

of the systematic blueshift of emission lines that is normally taken as clear evidence for dust formation in $\mathrm{SNe}$, and (2) this asymmetry is stronger at shorter wavelengths, as one expects if dust is responsible. Figure 2 shows the evolution of the $\mathrm{H} \alpha$ emission line during the first 6 months after discovery. Like SN 2006jc (Smith et al. 2008b), the systematic blueshift is present in the intermediate-width component, at velocities within $\pm 4000 \mathrm{~km} \mathrm{~s}^{-1}$ that arise in the post-shock region of CSM interaction. The profile is relatively symmetric at first, but as time proceeds the blue side of the line becomes much stronger than the red side (Figure 2). Also like in SN 2006jc, the line asymmetry is wavelength dependent (Figure 4).

The $\mathrm{H} \alpha$ equivalent width increases steadily during this time, from $150 \AA$ on 2010 November 5 to $1350 \AA$ on 2011 June 27 , and the maximum velocity of the wings of $\mathrm{H} \alpha$ increases from $3000 \mathrm{~km} \mathrm{~s}^{-1}$ to about $15,000 \mathrm{~km} \mathrm{~s}^{-1}$ during the same time. (Changes in the intrinsic line flux will be discussed in a future paper.) The characteristic continuum temperature around $7000 \mathrm{~K}$ stayed roughly constant during this time. Interestingly, the one obvious qualitative change in the spectrum is in the Ca II IR triplet around $8500 \AA$, which is absent in the earliest epochs, but then strengthens at later epochs. We return to the possible significance of this below. 


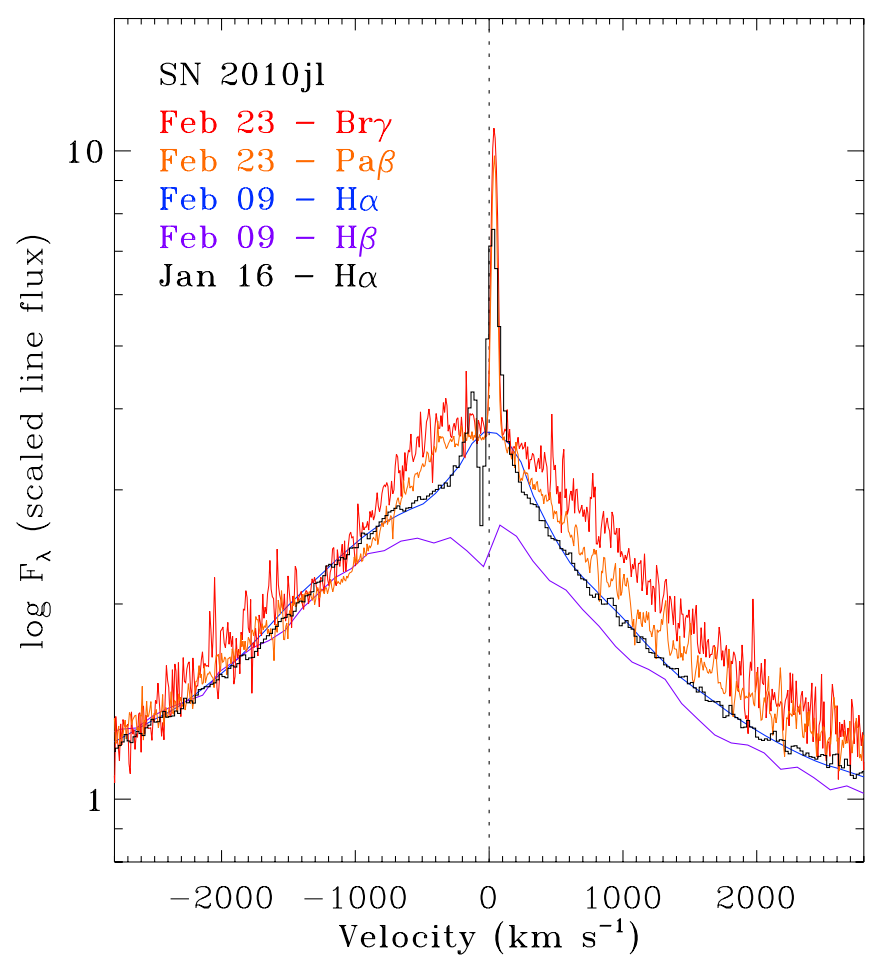

Figure 4. Wavelength dependence of the line-profile asymmetry in SN 2010j1. The H $\alpha$ profile from the 2011 January 16 MMT spectrum (black) is the same as in Figure 3. The $\mathrm{H} \beta$ (purple) and $\mathrm{H} \alpha$ (blue) profiles are from a single spectrum taken at Lick on 2011 February 9. The $\mathrm{H} \alpha$ profile did not change in the $\sim 3$ weeks between these observations (the difference at line center is due to the different spectral resolution used in the MMT and Lick spectra; see Table 1). The $\mathrm{Pa} \beta$ (orange) and $\mathrm{Br} \gamma$ (red) line profiles are from the FIRE spectrum taken 2 weeks later. These line profiles show that the asymmetry is more pronounced at shorter wavelengths, consistent with dust being the agent responsible for the blueshift of optical lines. The IR line profiles are less asymmetric, despite being taken after the optical lines shown here.

(A color version of this figure is available in the online journal.)

Figure 3 shows high-resolution MMT spectra of the $\mathrm{H} \alpha$ profile at three different epochs. Immediately after discovery, the intermediate-width component is dominated by a Lorentzian profile that may indicate electron scattering through high optical depths in the CSM (see, e.g., Smith et al. 2010), as we noted in our previous paper. A few months later, however, the intermediate-width component has qualitatively changed. The observed intermediate-width profile can be matched by adding a Gaussian with FWHM $=1000 \mathrm{~km} \mathrm{~s}^{-1}$ that is offset by $-700 \mathrm{~km} \mathrm{~s}^{-1}$ (solid magenta curve), in addition to the same Lorentzian. The relative strength of the Gaussian component (with the same width and offset) increases by the last epoch on 2011 June 27 (Figure 3).

The evolution of the narrow component of $\mathrm{H} \alpha$ arising in the pre-shock CSM is also interesting, shown in the inset of Figure 3. Although the red emission component appears relatively constant from 2010 November to 2011 January (note that these MMT spectra are taken with the same instrument configuration), the blueshifted $\mathrm{P}$ Cygni component, due to absorption by CSM along the line of sight, becomes stronger and shifts farther toward the blue. A similar effect was seen in the spectral evolution of SN 2006gy (Smith et al. 2010), which was taken as evidence for a Hubble-like expansion pattern in the CSM (i.e., faster expansion speeds at larger radii in the CSM). This, in turn, supported the hypothesis that the pre-SN CSM of SN 2006gy was formed in an LBV-like outburst rather than in a steady wind, and a similar scenario may apply to SN 2010jl. This appears to be consistent with the very massive progenitor star inferred for SN 2010jl (Smith et al. 2011b) and its dense, dusty CSM (Andrews et al. 2011). However, additional analysis of the CSM emission lines is needed.

\subsection{Interpretation}

If the formation of new dust is responsible for the systematic blueshift of the $\mathrm{H} \alpha$ line, then the new dust could reside in either the post-shock cooling zone or in the SN ejecta, since either could potentially block the receding side of the CSM interaction shock that produces the intermediate-width profiles (see Smith et al. 2008b). The time of dust formation is very soon after explosion, beginning after just 30 days. This is much sooner than the usual onset of dust formation in SNe, which typically occurs after a few hundred days. The hypothesis of post-shock dust formation therefore seems more likely, as only a dense radiative shock provides a mechanism for the rapid cooling.

Andrews et al. (2011) favor the interpretation that the IR excess they observe in SN 2010jl is due to an IR echo caused by pre-existing CSM dust that is warmed by the luminosity of the $\mathrm{SN}$, and not due to newly formed dust. They favor this interpretation based on the relatively cool dust temperatures of $T=$ $750 \mathrm{~K}$ in their model, whereas they expect newly formed dust to emit at $1600 \mathrm{~K}$. Andrews et al. (2011) did mention, however, that without a detailed study of the optical emission-line profiles, it is difficult to rule out the possibility of new dust formation as an explanation for the IR emission. As noted above, the $\mathrm{H} \alpha$ profile does in fact show very clear evidence of a systematic blueshift with time, which would normally be taken as strong evidence for new dust formation. The wavelength dependence of the asymmetry in the hydrogen line profiles from the blue to the IR seems to clinch the hypothesis that newly formed dust is responsible for the observed line asymmetry. This, however, seems to be at odds with the apparent dust temperatures.

High condensation temperatures around $1600 \mathrm{~K}$ are indeed appropriate for carbonaceous grains, as had been seen in $\mathrm{SN}$ 2006jc (Smith et al. 2008b). SN 2006jc was an SN Ibn, which might have involved an SN Ic expanding into an He-rich shell, so in that case C-rich dust seemed likely. However, some other dust species such as typical silicates have lower condensation temperatures closer to $1000 \mathrm{~K}$, and in SNe IIn we might expect O-rich grains like silicates, since the ejecta of SNe II are not necessarily strongly enriched with C. A lower assumed condensation temperature might allow the hypothesis of newly formed dust to be more consistent with observations. Moreover, we note that the single dust temperature model used by Andrews et al. somewhat underpredicts the observed $2 \mu \mathrm{m}$ flux (see their Figure 3), so perhaps there is room in the spectral energy distribution for some contribution from dust that is warmer than $750 \mathrm{~K}$. Our spectral observations show that much of the new dust causing asymmetric line profiles had already formed long before day 90, which is the time of the IR photometry analyzed by Andrews et al. (2011); perhaps some of that newly formed dust had already cooled by day 90 .

These details of the dust temperature aside, it is nevertheless possible that an IR echo produced much or most of the IR emission, as suggested by Andrews et al. (2011). While the line profiles presented here provide strong evidence that new dust formed in SN 2010jl, we do not know the mass or temperature of that new dust from the line profiles. It is therefore difficult from these observations to decide whether (1) new dust is solely responsible for the IR emission, or whether (2) new dust 
formed and dominates the line-profile asymmetry, but an IR echo from pre-existing circumstellar dust is still responsible for the observed IR excess at 3-5 $\mu \mathrm{m}$.

Option (1) would require that the newly formed dust cools from $1000 \mathrm{~K}$ to $750 \mathrm{~K}$ in about a month. This would require that most of the grains form in a brief episode before day 90, and then cool as the shell continues to expand and the grains come into temperature equilibrium with the post-shock gas. Careful study of this scenario is required before one can infer the expected temperature of newly formed dust. Option (2) would require that the newly formed dust is either less massive than the preexisting dust or somehow inefficient at radiating compared with the circumstellar dust, perhaps because it is located in optically thick clumps in the inhomogeneous post-shock cooling zone (e.g., Wooden et al. 1993). If option (2) is correct, it also implies that near- and mid-IR observations may not be reliable tracers of new dust formation in SNe.

\subsection{An Alternative Explanation?}

Since the formation of new dust based on asymmetric line profiles is seemingly at odds with the conclusion that most of the IR-emitting dust is pre-existing, it may be useful to consider alternative explanations for the blueshifted line profiles seen in SNe IIn. The intermediate-width lines in SNe IIn are produced in a physical region that is very different from line forming regions in normal SN ejecta, so careful consideration of possible sources of asymmetry are warranted. No alternative explanation has yet been proposed to account for the systematic blueshift in normal SNe or SNe IIn. We mention one speculative possibility here, which may follow from the extremely high optical depths and a consequent transition from optically thick to optically thin emission that occurs in SNe IIn (Smith et al. 2008a).

At early times, the symmetric Lorentzian line-profile shape seen in SNe IIn might not arise from the post-shock gas at all, but instead from an emission line formed in the slow preshock CSM that is broadened by electron scattering as the $\mathrm{H} \alpha$ line photons diffuse through the dense CSM ahead of the shock (Smith et al. 2010; Chugai 2001). In other words, the continuum photosphere is actually located ahead of the shock in the slow CSM, and the observed line wings do not trace the kinematics of the SN. Instead, the red wing of the line is an effect of electron scattering, and it is not directly related to redshifted gas on the receding side of the $\mathrm{SN}$.

Later on (after a few months in the case of SN 2010jl), the true intermediate-width component from the expanding post-shock gas is revealed once the pre-shock CSM becomes optically thin (i.e., the photosphere recedes back through the forward shock and exposes the expansion kinematics of the shock for the first time; see Smith et al. 2010). As the continuum photosphere recedes deeper into the post-shock zone or into the SN ejecta, it may then reveal $\mathrm{H} \alpha$ emission from the near side of the shock-but the continuum photosphere or dust may still block the far side of the post-shock shell, leading to the appearance of a blueshifted line profile. Interestingly, we note that the time when $\mathrm{H} \alpha$ develops asymmetric blueshifted profiles coincides roughly with the time when emission from the IR Ca II triplet strengthens (Figure 1). The onset of asymmetry also occurs when the line profile changes from Lorentzian (dominated by scattering) to predominantly Gaussian (Figure 3). Since the characteristic continuum temperature stays roughly constant (such that the growth in Ca II is not attributable to a change in temperature), this suggests that we are seeing deeper into the post-shock gas or SN ejecta at roughly the same time when the $\mathrm{H} \alpha$ line starts to become asymmetric.

In this case, the line profiles are already asymmetric and are "exposed" as the continuum scattering photosphere recedes through the forward shock. The asymmetric blueshift of the lines could be due to either extinction by newly formed dust in the post-shock shell, or to high continuum optical depths. In the case of SN 2010jl, we have presented IR spectra demonstrating that the effect is wavelength dependent, supporting the interpretation that dust is responsible in this object. However, other cases of SNe IIn may exist in which the wavelength dependence is not seen, and high continuum optical depths may be responsible for line asymmetry. We note that many SNe IIn remain luminous in the continuum for long periods of time after explosion, requiring the presence of high continuum optical depths. This ambiguity highlights the importance of obtaining spectra that span a wide wavelength range to look for reddening effects. Detailed radiative transfer simulations to investigate the feasibility of this effect would be valuable.

One additional test is that at much later times when the material is assured to be optically thin, the lines may remain asymmetric if dust is the responsible agent, whereas the profiles should become symmetric again if the continuum optical depth drops and the material becomes transparent. SN 2010jl is so bright that it may afford us the possibility to study its line profiles at very late times. Interestingly, we point out that the blueshifted $\mathrm{H} \alpha$ line profile in the SN IIn 2006tf persisted (and even became more pronounced) at very late times $\sim 445$ days after discovery (Smith et al. 2008a), suggesting perhaps that dust was indeed formed in the post-shock zones of that SN IIn as well.

The work presented here is based in part on observations made at the MMT Observatory, a joint facility of the Smithsonian Institution and the University of Arizona, and at the Lick Observatory, which is owned and operated by the University of California. Some of the data presented herein were obtained at the W. M. Keck Observatory, which is operated as a scientific partnership among the California Institute of Technology, the University of California, and NASA; the observatory was made possible by the generous financial support of the W. M. Keck Foundation. We thank the staffs at these observatories for their efficient assistance, as well as A. Barth, S. B. Cenko, J. Choi, A. Diamond-Stanic, R. J. Foley, K. Hiner, M. Kandrashoff, I. Kleiser, A. Merritt, J. Rex, and J. Walsh for their help with observations at Keck and Lick. The supernova research of A.V.F.'s group at U.C. Berkeley is supported by National Science Foundation grant AST-0908886 and by the TABASGO Foundation. J.M.S. is grateful to Marc J. Staley for a Graduate Fellowship.

Facilities: Keck:I (LRIS), Keck:II (DEIMOS), Shane (Kast), Magellan:Baade (FIRE).

\section{REFERENCES}

Andrews, J. E., Clayton, G. C., Wesson, R., et al. 2011, AJ, 142, 45

Andrews, J. E., Gallagher, J. S., Clayton, G. C., et al. 2010, ApJ, 715, 541

Benetti, S., Bufano, F., Vinko, J., et al. 2010, CBET, 2536, 1

Cherchneff, I., \& Dwek, E. 2010, ApJ, 713, 1

Chugai, N. N. 2001, MNRAS, 326, 1448

Colgan, S. W. J., Haas, M. R., Erickson, E. F., Lord, S. D., \& Hollenbach, D. J. 1994, ApJ, 427, 874

Danziger, I. J., Gouiffes, C., Bouchet, P., \& Lucy, L. B. 1989, IAU Circ., 4796, 1

Elmhamdi, A., Danziger, I. J., Chugai, N., et al. 2003, MNRAS, 338, 939 
Filippenko, A. V. 1982, PASP, 94, 715

Filippenko, A. V. 1997, ARA\&A, 35, 309

Fox, O. D., Chevalier, R. A., Skrutskie, M. F., et al. 2011, ApJ, 741, 7

Fox, O., Skrutskie, M. F., Chevalier, R. A., et al. 2009, ApJ, 691, 650

Gehrz, R. D., \& Ney, E. P. 1989, Proc. Nat. Acad. Sci., 87, 435

Kotak, R., Meikle, W. P. S., Farrah, D., et al. 2009, ApJ, 704, 306

Li, W., Leaman, J., Chornock, R., et al. 2011, MNRAS, 412, 1441

Lucy, L. B., Danziger, I. J., Gouiffes, G., \& Bouchet, P. 1989, in Structure and Dynamics of the Interstellar Medium, ed. G. Tenorio-Tagle et al. (Berlin: Springer), 164

Matilla, S., Meikle, W. P. S., Lundqvist, P., et al. 2008, MNRAS, 389, 141

Matsuura, M., Dwek, E., Meixner, M., et al. 2011, Science, 333, 1258

Meikle, W. P. S., Kotak, R., Farrah, D., et al. 2011, ApJ, 732, 109

Meikle, W. P. S., Mattila, S., Pastorello, A., et al. 2007, ApJ, 665, 608

Miller, J. S., \& Stone, R. P. S. 1993, Lick Obs. Tech. Rep. 66 (Santa Cruz, CA: Lick Obs.)
Newton, J., \& Puckett, T. 2010, CBET, 2532, 1

Patat, F., Taubenberger, S., Benetti, S., Pastorello, A., \& Harutyunyan, A. 2011, A\&A, 527, L6

Pozzo, M., Meikle, W. P. S., Fassia, A., et al. 2004, MNRAS, 352, 457

Smith, N., Chornock, R., Li, W., et al. 2008a, ApJ, 686, 467

Smith, N., Chornock, R., Silverman, J. M., Filippenko, A. V., \& Foley, R. J. 2010, ApJ, 709, 856

Smith, N., Foley, R. J., \& Filippenko, A. V. 2008b, ApJ, 680, 568

Smith, N., Li, W., Filippenko, A. V., \& Chornock, R. 2011a, MNRAS, 412, 1522

Smith, N., Li, W., Miller, A. A., et al. 2011b, ApJ, 732, 63

Smith, N., Silverman, J. M., Chornock, R., et al. 2009, ApJ, 695, 1334

Sugerman, B. E. K., Ercolano, B., Barlow, M. J., et al. 2006, Science, 313, 196

Szalai, R., Vinko, J., Balog, Z., et al. 2011, A\&A, 527, A61

Wooden, D. H., Rank, D. M., Bregman, J. D., et al. 1993, ApJS, 88, 477 